Check for updates

Cite this: Mater. Adv., 2021, 2,3954

Received 22nd December 2020, Accepted 8th May 2021

DOI: 10.1039/d0ma01008a

rsc.li/materials-advances

\section{Investigation of the influence of natural deep eutectic solvents (NaDES) in the properties of chitosan-stabilised films $\dagger$}

\author{
Antonella Rozaria Nefeli Pontillo, ${ }^{a}$ Spyridon Koutsoukos, (DD ${ }^{b}$ Tom Welton (D) ${ }^{b}$ and \\ Anastasia Detsi*a
}

\begin{abstract}
In the present work, the plasticising effect of the natural deep eutectic solvents (NaDESs) choline chloride/lactic acid and betaine/lactic acid in the formation of chitosan-stabilised films is investigated. The films were prepared by the casting technique, dissolving chitosan in $1 \% \mathrm{w} / \mathrm{v}$ solution of choline chloride and lactic acid or betaine and lactic acid in water, either as a pre-formed NaDES or as separately added components (physical mixture), without the addition of acetic acid. The physicochemical and mechanical properties of the formed films were studied. High concentration of the NADES (83\%) increases the weight and thickness of the films. The reference chitosan films containing acetic acid or lactic acid are inelastic and very resistant to burst. On the other hand, the addition of betaine increases significantly the elongation capacity of the film prior to burst. The water vapour permeability of the films prepared from the NaDES solutions presents a 4.5 and a 2.6 -fold increase, respectively, in comparison with acetic acid containing films. The materials prepared using the NaDESs have distinct differences to those prepared using the physical mixture of the NADESs' components, as shown from the thermal and FT-IR spectroscopic analyses data. Finally, SEM imaging revealed differences among the surfaces of the films.
\end{abstract}

\section{Introduction}

Deep Eutectic Solvents (DES) are mixtures of two or more components, a hydrogen bond acceptor and a hydrogen bond donor, with a low-temperature eutectic point. When the components of the DES are naturally occurring compounds, the solvents are characterised as Natural Deep Eutectic Solvents (NaDES). They constitute a transparent, usually viscous, liquid at room temperature with significantly low melting point and negligible vapour pressure. Due to their high biodegradability potential, NaDESs have emerged in research towards greener processes development. Their applications as extraction solvents, catalysis agents in synthetic processes, or plasticisers during the production of thermoplastic films are being intensively investigated. $^{1-9}$

\footnotetext{
${ }^{a}$ Laboratory of Organic Chemistry, Department of Chemical Sciences, School of Chemical Engineering, National Technical University of Athens, Zografou, Greece. E-mail: adetsi@chemeng.ntua.gr

${ }^{b}$ Department of Chemistry, Molecular Sciences Research Hub, Imperial College London, White City Campus, London W12 OBZ, UK

$\dagger$ Electronic supplementary information (ESI) available: $\mathrm{pH}$ curves of the NaDESs aqueous solutions, FT-IR spectra and TGA graphs of the NaDESs, deconvoluted FT-IR spectra of the films, TGA-MS of the films and the starting materials. See DOI: $10.1039 /$ d0ma01008a
}

The gelation of NaDESs has only recently been reported and proposed for a variety of different applications. Xia et al. ${ }^{10}$ investigated choline chloride-xylitol NaDES, employing xanthan gum as the gelation agent. The ability of the NaDES to dissolve bioactive compounds like quercetin, renders the so-called eutectogels as a promising alternative for oral and transdermal drug delivery. Marullo et al. ${ }^{11}$ prepared choline chloride-phenylacetic acid eutectogels using different amino acids as gelators. The study revealed that the prepared formulations could be used as sorbents for the removal of dyes from wastewater with high removal efficiency and recyclability.

Chitosan exhibits strong antimicrobial and antifungal activity. ${ }^{12,13}$ Due to their inherent non-toxic and biodegradable nature, chitosan films are considered as versatile alternatives to conventional plastic polymer films, which could find use in numerous applications. In the food industry, chitosan films have been investigated as packaging material to prolong the shelf-life of products. ${ }^{14}$ In the pharmaceutical industry, their use as wound dressings is being investigated, as they could incorporate a therapeutic agent offering a controlled release and enhancing its properties. ${ }^{15,16}$

The mechanical properties such as the elasticity and tear resistance of different films can be varied significantly, depending on the requirements of each application. Flexibility and pliability are essential properties for wound dressings in order to cover the 
wound and prevent infection. ${ }^{17,18}$ On the other hand, a packaging material may be flexible or inelastic to maintain its integrity throughout the transportation and storage of the products. ${ }^{19,20}$

Water vapour permeability (WVP) is a significant property of films. Water vapours transfer through a film by absorption, diffusion through the film due to interaction with the polymer and desorption. ${ }^{21}$ All films are permeable, but depending on their structure, some may create a stronger barrier. The requirements of the application define the selection of polymers and additives. For instance, in food packaging, research is oriented towards decreasing the permeability of water through the film, thus protecting the shelf life of packaged products, as water causes bacterial development and, consequently, spoilage of the aliments. $^{22-24}$ On the other hand, the permeability of water through wound dressings should not be too low as the healing time and the risk of bacterial infection increases, ${ }^{25,26}$ nor too high as it may cause dehydration of the area and subsequently, a scar. ${ }^{27,28}$

In spite of its remarkable bioactivity and extent of potential applications, chitosan presents an inherent difficulty to dissolve in any solvent apart from acidic aqueous solutions (most commonly $1 \%$ acetic acid). In an effort to explore additional options regarding chitosan's solubility and film-making ability, in the present work, we investigated the solubility of chitosan in aqueous solution of two selected NaDESs namely choline chloride:lactic acid ( $\mathrm{ChCl}: \mathrm{LA} 1: 1.5)$ and betaine:lactic acid (bet: LA 1:2) and demonstrated that chitosan can be dissolved in solutions of $1 \% \mathrm{w} / \mathrm{v}$ NaDESs in water and, thus, elastic films were prepared via the casting technique.

According to the experimental and computational work of Gutiérrez et al., ${ }^{29}$ large dilutions of a NaDES disrupt the intermolecular interactions between the components. Therefore, it is theoretically expected that a $1 \%$ aqueous solution of a NaDES is equivalent to an aqueous mixture of its components.

In order to comprehend whether the pre-formation of the NaDES has an impact on properties of the films, two different films were prepared for each NaDES. For the first film, the NaDES was synthesised and dissolved in water, while for the second film, the appropriate amounts of the two components were directly dissolved in water, prior to chitosan addition. Subsequently, the water was evaporated, forming the following films: F/ChCl:LA NADES and F/ChCl:LA mix. and F/bet:LA NaDES and F/bet:LA mix., respectively. The addition of acetic acid was not required in any of the films, as the formed aqueous solution was already acidic.

Moreover, the effect of lactic acid (LA) was also monitored in two different concentrations, first $0.4 \%$ (F/0.4LA), equivalent to the concentration of LA in 1\% ChCl:LA NaDES, and 0.5\% (F/0.5LA) equivalent to the LA in 1\% of the bet:LA NaDES. Finally, the results were compared with films produced with the addition of the wellknown plasticiser Tween 80 (F/Tween).

\section{Results and discussion}

After their preparation, distinct differences were observed among the films. All films were compact, and predominantly off-white but their texture and elasticity varied significantly. Visual observation revealed that the F/AA was the most rigid film, while the films containing NaDES or the physical mixtures were more elastic and pliable. No noticeable difference occurred when different lactic acid concentrations were used, but they were both significantly more elastic than the F/AA. The presence of Tween 80 led to the formation of a non-transparent film and added elasticity to the film. Finally, the two choline chloride containing films were highly hydrophilic. The $\mathrm{pH}$ of all the film-forming solutions was measured and found to be between $2.84 \pm 0.04$ and $3.25 \pm 0.04$.

\section{Weight and thickness}

The chemical structure and the size of the acid plays a significant role in the mechanical and physicochemical properties of the films. ${ }^{35}$ Therefore, the films prepared from solutions of lactic acid, which is an alpha-hydroxy acid, were found to be thicker than the films formed by the solubilisation of chitosan in acetic acid. The use of common plasticizers such as Tween 80 , enlarges the spacing between the chains of the polymer, ${ }^{36}$ so the observed difference in the thickness of the F/Tween film and the F/AA film was expected (Table 1).

The films prepared from the NaDES solutions (F/bet:LA NaDES and F/ChCl:LA NaDES) are significantly thicker than the corresponding films prepared from the LA solution (F/0.5LA and F/0.4LA). A plausible explanation is that the presence of the betaine or the choline chloride moiety provides an additional spacing between the chitosan chains as these molecules can form additional hydrogen bonds and electrostatic interactions with the polymer functional groups which results in the swelling of the film.

On the other hand, the NaDES-containing films appear to be only slightly thicker than the films containing the components of the NaDES when they are added as a mixture.

\section{Burst strength (BS) and distance at burst (DB)}

The measurements of the resistance on burst (burst strength, BS) and total elongation of the films before burst (distance at burst, DB), are presented in Fig. 1. BS of the F/AA was measured to be $5.7 \mathrm{~N}$, which with the addition of the plasticiser Tween 80 (F/Tween) is further increased $(7.0 \mathrm{~N})$.

The impact of the acid on the films' mechanical properties was again confirmed: the presence of lactic acid instead of acetic acid increases both the BS and the DB of the films. Moreover, a higher concentration of lactic acid has a strong

Table 1 Weight $(\mathrm{mg})$ and thickness $(\mathrm{mm})$ of the prepared films

\begin{tabular}{lll}
\hline Film & Weight $(\mathrm{mg})$ & Thickness $(\mathrm{mm})$ \\
\hline F/AA & $113.76 \pm 4.3$ & $0.037 \pm 0.005$ \\
F/Tween & $183.04 \pm 6.8$ & $0.081 \pm 0.020$ \\
F/bet:LA NaDES & $495.40 \pm 15.0$ & $0.152 \pm 0.070$ \\
F/bet:LA mix. & $465.63 \pm 9.7$ & $0.110 \pm 0.010$ \\
F/0.5LA & $217.70 \pm 16.8$ & $0.057 \pm 0.020$ \\
F/ChCl:LA NaDES & $518.76 \pm 9.4$ & $0.126 \pm 0.019$ \\
F/ChCl:LA mix. & $487.33 \pm 15.5$ & $0.105 \pm 0.003$ \\
F/0.4LA & $209.45 \pm 11.2$ & $0.052 \pm 0.020$
\end{tabular}




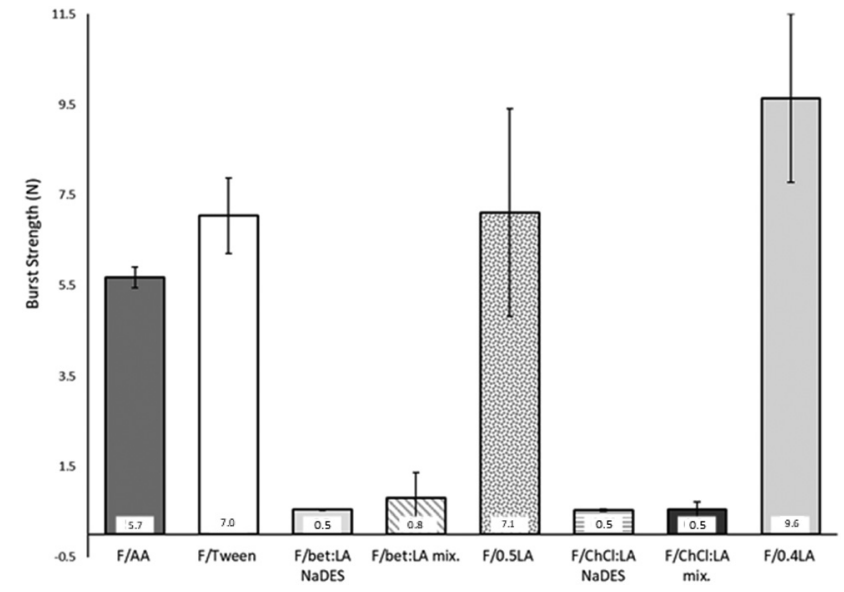

Fig. 1 Burst strength results of the different films.

effect on the BS of the films, while the DB remains practically the same. Therefore, the lactic acid containing films (F/0.5LA and F/0.4LA) are more elastic, yet more difficult to break than the acetic acid film (F/AA). This observation is in accordance to the work of Caner $e t a l .{ }^{21}$ On the other hand, the films prepared from the NaDES solutions (F/bet:LA NaDES and F/ChCl:LA NaDES) presented significantly lower resistance to rupture, but were more elastic compared to the reference films. The films formed from the NaDES solution or from the solution containing the mixture of the two components of the NaDES, required $86-95 \%$ less burst strength to be ruptured while their ability to elongate was $13-78 \%$ higher than the corresponding films formed from the solutions of the acids.

The plasticising effect achieved in the films F/bet:LA NaDES, F/bet:LA mix., F/ChCl:LA NaDES and F/ChCl:LA mix. is clearly observed in Fig. 2. Higher elasticity is expected with the addition of NaDESs, as the free space between the polymeric chains is increased. ${ }^{37}$ Hence, the DB of F/bet:LA NaDES and F/ChCl:LA NaDES is increased to $20.1 \mathrm{~mm}$ and $11.0 \mathrm{~mm}$ respectively while for F/AA is $4.8 \mathrm{~mm}$.

Moreover, the $\mathrm{F} / \mathrm{bet}$ :LA mix. and $\mathrm{F} / \mathrm{ChCl}$ :LA mix. presented similar elongation ability prior to burst as the F/bet:LA NaDES

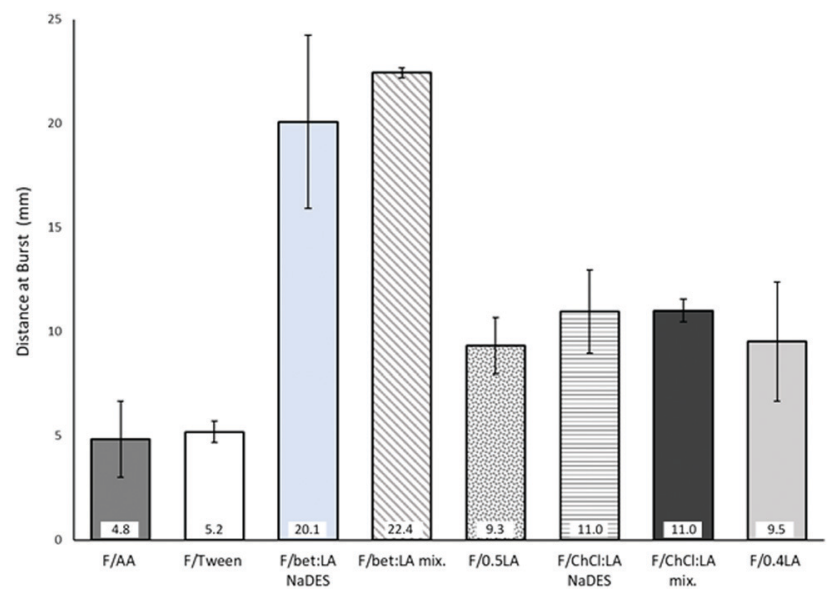

Fig. 2 Distance at burst (b) results of the different films. and $\mathrm{F} / \mathrm{ChCl}$ :LA NaDES respectively, suggesting that the addition of the two components separately or as a pre-formed NaDES does not affect the mechanical properties of the films.

Another interesting observation is that both betaine containing films were noticeably more elastic than F/ChCl:LA NaDES and F/ChCl:LA mix.

\section{Water vapour permeability}

WVP measurements showed that the F/AA, F/0.5LA and F/0.4LA almost equally permit the water vapours to pass through. The presence of any additional plasticiser or solvent significantly increases the WVP of the films.

The highly hydrophilic character of choline chloride promotes the water vapour diffusion. Therefore, the choline chloride containing films exhibit the highest WVP. ${ }^{37}$

As can be observed from Fig. 3, when the two components of the NaDES are added separately in the film forming solution, the WVP is lower compared to the films where the NaDES was synthesised prior to the chitosan dissolution. A plausible explanation could be that when the components of the NaDES are separately added, they are more uniformly incorporated between the chitosan chains. This could be attributed to the hydrogen bonds of the NaDES, resulting in higher swelling of the polymer, enlarging the spacing formed between the polymer chains and permitting the permeant water vapour to pass through more easily. If the existence of NaDES causes the formation of hydrogen-bonded, water-friendly, clusters between the chitosan chains, then these clusters could easily act as passages for water to enter the film WVP of aluminum foil, a strong barrier of water, was measured as reference.

\section{FT-IR}

In the FT-IR spectra of the F/AA (Fig. 4), the following peaks appear: a broad medium peak at $3405 \mathrm{~cm}^{-1}(\mathrm{OH}$ and $\mathrm{NH}$ stretch), a strong double peak at 2924 and $2859 \mathrm{~cm}^{-1}$ (C-H stretching vibration, $-\mathrm{CH}_{3}$ ), a low intensity peak at $1735 \mathrm{~cm}^{-1}$ ( $\mathrm{C}=\mathrm{O}$ stretching), $1643 \mathrm{~cm}^{-1}$ (amide I band of the polymer), $1548 \mathrm{~cm}^{-1}$ (antisymmetric $-\mathrm{COO}^{-}$stretching vibration) and at $1374 \mathrm{~cm}^{-1}$ (symmetric $-\mathrm{COO}^{-}$stretching vibration). The low intensity of both the $\mathrm{C}=\mathrm{O}$ and the $-\mathrm{OH}$ peaks, indicates the existence of chitosan acetate and that only a minimal amount

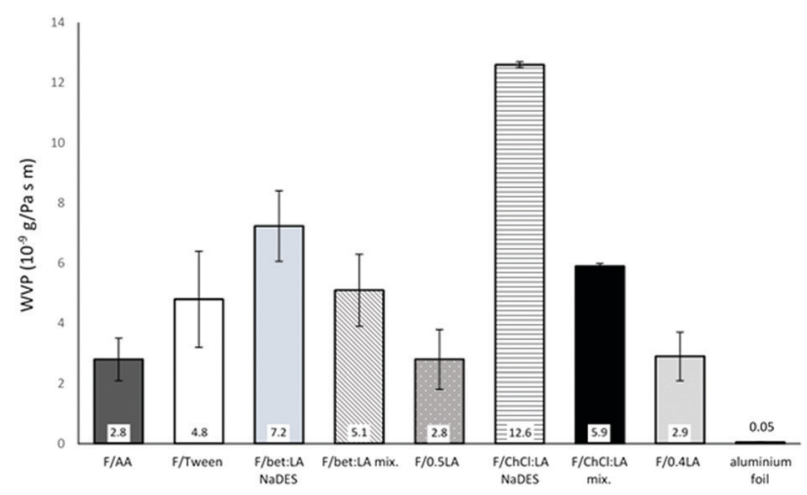

Fig. 3 Water vapour permeability of all films. 


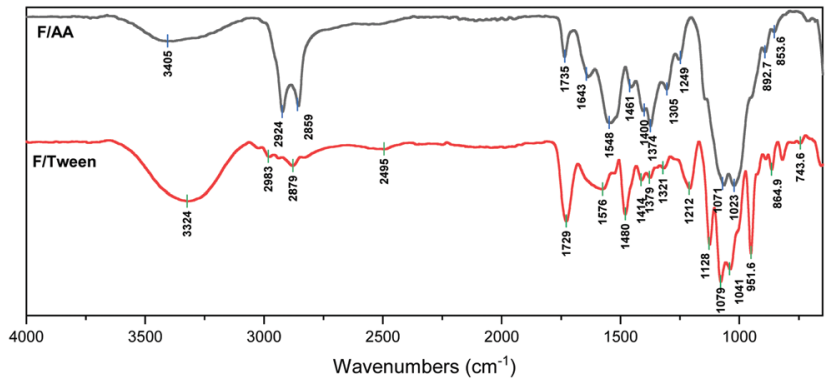

Fig. 4 FT-IR spectra of the F/AA (black) and F/Tween (red).

of free acid remains bound in the film, the rest is evaporated during the incubation period. ${ }^{38,39}$

The spectrum of F/Tween (Fig. 4) reveals that Tween 80 does not chemically interact with chitosan, as there are only minor peak shifts compared to the neat Tween 80 spectrum. $^{40}$

From the spectra of F/0.4LA and F/0.5LA (Fig. 5) it can be observed that the peaks in the range 3600 to $3000 \mathrm{~cm}^{-1}$, as well as the peaks from the $\mathrm{C}=\mathrm{O}$ stretching vibration of the $\mathrm{COOH}$ group (present at $1721 \mathrm{~cm}^{-1}$ in the spectrum of $\mathrm{F} / 0.4 \mathrm{LA}$ and at $1722 \mathrm{~cm}^{-1}$ in the spectrum of F/0.5LA) are significantly more intense than in the spectrum of F/AA. This indicates that the excess of lactic acid remains in the system in its neutral form, as it is less volatile than the acetic acid. The two bands at $2982 \mathrm{~cm}^{-1}$ ( $\mathrm{CH}_{2}$ vibrations) and $2521 \mathrm{~cm}^{-1}$ (-OH stretching) correspond to the lactic acid. Moreover, the band at $1566 \mathrm{~cm}^{-1}$ or $1570 \mathrm{~cm}^{-1}$, respectively, is attributed to the antisymmetric $-\mathrm{COO}^{-}$stretching vibration of the lactate ion, which has been shifted due to the presence of the electron-withdrawing - $-\mathrm{OH}$ group in the 2-position. Furthermore, a new small shoulder appears at $1521 \mathrm{~cm}^{-1}$, corresponding to the ammonium cation, verifying the formation of chitosan lactate. ${ }^{41-43}$

In the spectrum of F/bet:LA NaDES (Fig. 6) the bands at 2923 and $2854 \mathrm{~cm}^{-1}$ are very strong, while the $3412 \mathrm{~cm}^{-1}$ peak is significantly weaker and the $-\mathrm{OH}$ peak is negligible, indicative of a smaller amount of the acid in its neutral form. Moreover, the $-\mathrm{COOH}$ and the $-\mathrm{COO}^{-}$peaks are now reversely intense, as the carboxyl groups are consumed, indicating the formation of lactate ion.

For the F/bet:LA mix. (Fig. 6) the peak at $1727 \mathrm{~cm}^{-1}$ is of significantly lower intensity than the $1619 \mathrm{~cm}^{-1}$ peak, indicative of a greater amount of lactic acid in its neutral form compared to the F/bet:LA. ${ }^{44,45}$

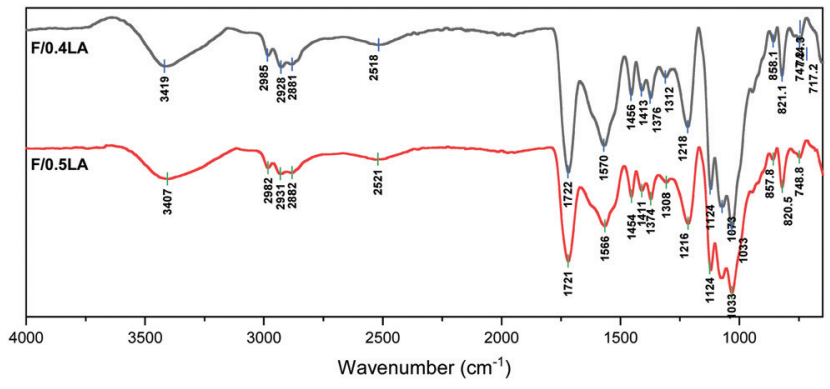

Fig. 5 FT-IR spectra of the F/0.4LA (black) and F/0.5LA (red).

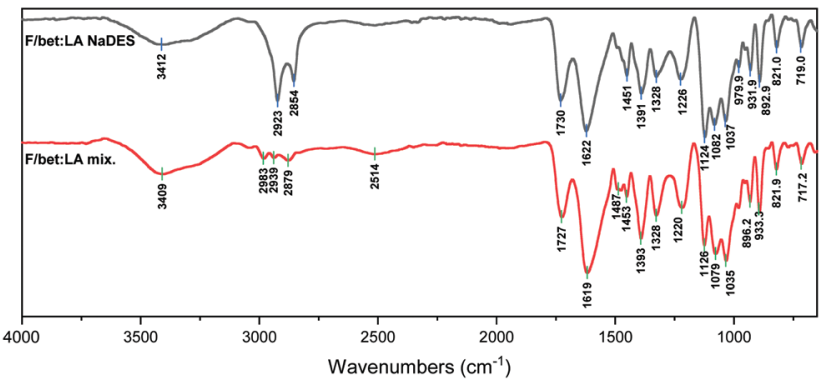

Fig. 6 FT-IR spectra of the F/bet:LA NaDES (black) and the F/bet:LA mix. (red).

The FT-IR spectra of F/ChCl:LA NaDES and F/ChCl:LA mix. (Fig. 7) present broad strong peak at around $3323 \mathrm{~cm}^{-1}$ and $3323 \mathrm{~cm}^{-1}$ respectively attributed to the $\mathrm{O}-\mathrm{H}$ and $\mathrm{N}-\mathrm{H}$ stretching vibrations. The absorbance from the carboxyl group of LA is observed at $1731 \mathrm{~cm}^{-1}$ in the $\mathrm{F} / \mathrm{ChCl}$ :LA NaDES spectrum and is shifted by $2 \mathrm{~cm}^{-1}$ in the F/ChCl:LA mix. spectrum. The absorbances at 1479 and $1480 \mathrm{~cm}^{-1}$, respectively, are attributed to $-\mathrm{CH}_{2}$ scissoring, and the peaks at 1127 and $1128 \mathrm{~cm}^{-1}$ are attributed to the $\mathrm{C}-\mathrm{C}$ stretching vibration. ${ }^{43,46}$

The FT-IR spectra of the NaDESs bet:LA and ChCl:LA are presented in Fig. S2 (ESI $\dagger$ ).

All samples present a wide set of peaks in the range 1200$1000 \mathrm{~cm}^{-1}$, with different behaviour for each sample. These peaks have been deconvoluted, by fitting Gaussian functions, in order to reveal overlapping peaks (Fig. S3-S9, ESI $\dagger$ ) while the Gauss Curve equation parameters are explained in Fig. S10 (ESI $\dagger$ ). The accuracy of the Gaussian fitting can be seen at Tables S1-S7 in the ESI. $\dagger$ Table 2 summarises the revealed peaks of all samples in the studied range.

From Table 2, it can be seen that in both samples containing acetic acid, a peak at $1150 / 1149 \mathrm{~cm}^{-1}$ appears, corresponding to the $\mathrm{C}-\mathrm{C}$ stretching vibration of the acid (Peak 1). However, when lactic acid is present in a film, the band is shifted towards lower wavenumbers. For the F/Tween, both bands appear, Peak 1 corresponding to the acid and Peak 2 corresponding to the plasticiser. Peak $3(\mathrm{C}-\mathrm{O})$ appears in all samples as well as in the raw chitosan spectrum. Peak 4, is characteristic of the lactic acid, although slightly shifted from that reported in literature $\left(1043 \mathrm{~cm}^{-1}\right)$. Peak 5 differs among the studied samples, probably because it is due to different interactions. For F/bet:LA

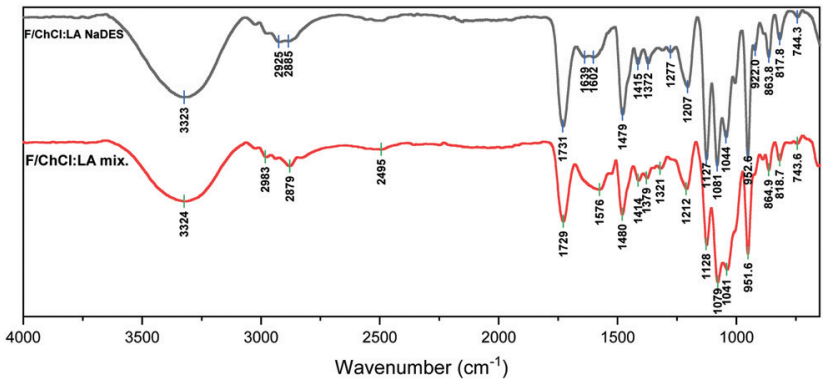

Fig. 7 FT-IR spectra of the F/ChCl:LA NaDES (top) and the F/ChCl:LA mix. (bottom) 
Table 2 Deconvolution of the most important bands in the FT-IR spectra of the samples in the range $1200-1000 \mathrm{~cm}^{-1}$

\begin{tabular}{llllll}
\hline Sample & $\begin{array}{l}\text { Peak 1 } \\
\left(\mathrm{cm}^{-1}\right)\end{array}$ & $\begin{array}{l}\text { Peak 2 } \\
\left(\mathrm{cm}^{-1}\right)\end{array}$ & $\begin{array}{l}\text { Peak 3 } \\
\left(\mathrm{cm}^{-1}\right)\end{array}$ & $\begin{array}{l}\text { Peak 4 } \\
\left(\mathrm{cm}^{-1}\right)\end{array}$ & $\begin{array}{l}\text { Peak 5 } \\
\left(\mathrm{cm}^{-1}\right)\end{array}$ \\
\hline F/AA & 1150 & - & 1076 & - & - \\
F/Tween & 1149 & 1127 & 1078 & 1024 & - \\
F/O.5LA & - & 1124 & 1079 & 1030 & 1001 \\
F/bet:LA NaDES & - & 1126 & 1082 & 1035 & 1015 \\
F/bet:LA mix. & - & 1127 & 1079 & 1030 & 1001 \\
F/ChCl:LA NaDES & - & 1126 & 1082 & 1043 & 1004 \\
F/ChCl:LA mix. & - & 1126 & 1082 & 1040 & 998
\end{tabular}

NaDES and F/bet:LA mix. Peak 5 corresponds to the R-N $\left(\mathrm{CH}_{3}\right)_{3}{ }^{+}$ skeletal vibrations, while for F/0.5LA it corresponds to $\mathrm{C}-\mathrm{H}$ or $\mathrm{C}-\mathrm{O}$ vibrations (rocking, deformation, stretching).

The interesting observation in this range, is that the relative intensities of the peaks differ between samples. For F/bet:LA NaDES Peak 2 is the strongest, while in F/bet:LA mix. and F/ 0.4LA the Peaks 3 and 4 are stronger than Peak 2 . This could be explained with a higher amount of lactic acid existing in its neutral form, thus, it could be supposed that the salt formation is hindered in the case of F/bet:LA NaDES. This could also be verified by the relative intensities of the 1730 and $1622 \mathrm{~cm}^{-1}$ peaks (1727 and $1619 \mathrm{~cm}^{-1}$, respectively, in the mixture): in $\mathrm{F} /$ bet:LA NaDES the difference in intensity is much smaller than in F/bet:LA mix., although the amounts of each component are the same.

Similarly, the comparison of the relative intensity of Peaks 2, 3 and 4 between the F/ChCl:LA NaDES and F/ChCl:LA mix. spectra indicates the lower amount of free lactic acid in the former sample.

Having established that the interaction between chitosan and each acid occurs towards the formation of the corresponding salt, it has been confirmed that the excess of acetic acid evaporates during the film formation while lactic acid remains in the system. Furthermore, the addition of lactic acid as a component of a preformed NaDES into the system 'protects' the lactic acid in its neutral form. This results in the reaction with fewer amino groups of chitosan in contrast to the mixture of the components, in which the expected chemical equilibrium is maintained. Therefore, there is an indication of different chemical environments existing in the samples, which could be responsible for the differences observed in the thermal and mechanical properties of the films.

\section{TGA-MS}

Thermal degradation of the two studied NaDES (Fig. S11, ESI $\dagger$ ) follows a simple profile, similar to other DESs in the literature. ${ }^{46,47}$ The decomposition happens in two steps: LA starts decomposing first, followed by the decomposition of the hydrogen bond acceptor (HBA), at similar temperatures $\left(192{ }^{\circ} \mathrm{C}\right.$ for bet:LA and $194^{\circ} \mathrm{C}$ for ChCl:LA), which indicates that the use of betaine or choline chloride doesn't affect the thermal properties of the NaDES. Bet:LA has greater carbon residue compared to ChCl:LA (3.4\% over $1.2 \%$ ), which could be attributed to the different decomposition pathway of the carboxyl over the hydroxyl group on the HBA. The results of the TGA-MS
Table 3 Summary table of the TGA moisture content, $T_{\text {onset, }} T_{\text {max.rate, }}, T_{1 / 2}$ residue of the films and the NaDESs

\begin{tabular}{llllll}
\hline Sample & $\begin{array}{l}\text { TGA moisture } \\
\text { content (\% weight) }\end{array}$ & $\begin{array}{l}T_{\text {onset }}\left({ }^{\circ} \mathrm{C}\right) \\
\left(\begin{array}{l}T_{\text {max.rate }} \\
\left({ }^{\circ} \mathrm{C}\right)\end{array}\right.\end{array}$ & $\begin{array}{l}T_{1 / 2} \\
\left({ }^{\circ} \mathrm{C}\right)\end{array}$ & $\begin{array}{l}\text { Residue } \\
(\% \text { weight })\end{array}$ \\
\hline bet:LA NaDES & 4.4 & 192 & 249 & 243 & 3.4 \\
ChCl:LA NaDES & 5.5 & 194 & 262 & 253 & 1.2 \\
F/AA & 9.1 & 200 & 278 & 280 & 25.2 \\
F/Tween & 5.0 & 247 & 429 & 380 & 11.4 \\
F/bet:LA NaDES & 6.3 & 201 & 250 & 249 & 6.5 \\
F/bet:LA mix. & 6.4 & 211 & 255 & 252 & 1.0 \\
F/0.5LA & 4.8 & 163 & 178,283 & 261 & 12.0 \\
F/ChCl:LA NaDES & 8.2 & 225 & 268 & 262 & 5.1 \\
F/ChCl:LA mix. & 8.3 & 209 & 273 & 258 & 3.6 \\
Chitosan & 3.0 & 272 & 294 & 299 & 36 \\
Acetic acid & - & - & - & - & - \\
Lactic acid & 12.9 & 145 & 179 & 188 & 0.3 \\
Betaine & 0.9 & 295 & 302 & 302 & 1.8 \\
Choline chloride & 1.8 & 303 & 307 & 307 & 1.0
\end{tabular}

measurements of the two NaDESs, as well as of all films and components, are presented in Table 3, while the corresponding spectra are presented in Fig. S12 and S23 (ESI $\dagger$ ).

F/AA shows decomposition throughout the heating range $\left(T_{\text {onset }}=200{ }^{\circ} \mathrm{C}\right)$, which results in $25 \mathrm{wt} \%$ carbon residue (Fig. S12, ESI $\dagger$ ). As expected, chitosan decomposes mainly to $\mathrm{H}_{2} \mathrm{O}(\mathrm{m} / \mathrm{z}=18)$ and $\mathrm{CO}_{2}(\mathrm{~m} / \mathrm{z}=44)$. This can be confirmed from the MS spectra of the neat polymer (Fig. S19, ESI $\dagger$ ). Spectra with $m / z=16$ and 32, which correspond to atomic and molecular oxygen, show a continuous reduction in intensity, as the MS column is flushed with $\mathrm{N}_{2}$ through the measurement, removing the trapped oxygen. No oxygen seems to be produced during the decomposition of chitosan. The graph for $\mathrm{m} / \mathrm{z}=29$ is interesting, as it shows an initial reduction, followed by a constant production in the range $300-500{ }^{\circ} \mathrm{C}$, which indicates that although there was an initial production of this mass fragment, the main decomposition happens in this range. The $m / z=29$ fragment could be attributed to the ${ }^{+} \mathrm{HC} \mathrm{NH}_{2}$ fragment of the chitosan ring. As the acetic acid evaporates during instrument equilibration time and does not show any characteristic MS peaks (Fig. S20, ESI $\dagger$ ), the resemblance of the F/AA spectrum to that of pure chitosan is expected.

The TGA of the F/Tween sample shows a higher decomposition starting point $\left(T_{\text {onset }}=247^{\circ} \mathrm{C}\right)$ compared to F/AA leading to significantly lower carbon residue (11 wt\%) (Fig. S13, ESI $\dagger$ ). It seems that the plasticiser has a double role, at lower temperatures it provides the film with thermal resistance, while at higher temperatures it promotes a cleaner burning of the film, leading to lower carbon residue.

The MS of F/Tween shows that the production of $m / z=29$ is more apparent and also a product with $\mathrm{m} / \mathrm{z}=14$ is released. The latter could be attributed to $\mathrm{CH}_{2}{ }^{+}$released by the decomposition of Tween 80 . The increase of $m / z=29$ products could again be attributed to Tween, as $\mathrm{CHO}^{+} \cdot$ and $\mathrm{C}_{2} \mathrm{H}_{5}^{+}$fragments have identical masses. Also, around $450{ }^{\circ} \mathrm{C}$ a rapid production of atomic oxygen is observed, presumably due to the decomposition of Tween's side chains.

F/0.5LA shows a significantly lower decomposition point $\left(T_{\text {onset }}=167^{\circ} \mathrm{C}\right)$ compared to F/AA (Fig. S14, ESI $\dagger$ ). This can 
be explained by considering the presence of excess of lactic acid, which unlike acetic acid, remains physically bound to the film due to its lack of volatility. MS profile reveals the constant production of $\mathrm{H}_{2} \mathrm{O}$ throughout the temperature range, probably due to the initial decomposition of excess lactic acid. The TGAMS spectrum of lactic acid (Fig. S21, ESI $\dagger$ ) confirms the decomposition of the acid at a significantly lower temperature $\left(T_{\text {onset }}=145{ }^{\circ} \mathrm{C}\right)$. It is therefore possible that polymer-bound LA is released stepwise and decomposes over a larger temperature range.

TGA of F/bet:LA NaDES (Fig. S15, ESI $\dagger$ ) is in general similar to bet:LA NaDES (Fig. S11, ESI $\dagger$ ). The initial decomposition point has slightly shifted to higher temperature $\left(T_{\text {onset }}=201{ }^{\circ} \mathrm{C}\right)$ compared to the NaDES $\left(192{ }^{\circ} \mathrm{C}\right)$. The carbon residue is almost doubled (from 3.6 to $6.5 \mathrm{wt} \%$ ), due to the presence of chitosan. According to the synthetic procedure, a NaDES film consists of $83.3 \mathrm{wt} \%$ NaDES and $16.7 \%$ chitosan. Taking into account the carbon residue of bet:LA NaDES (3.4\%) and F/AA $(25.2 \%)$, as well as their relative ratios in the NaDES film, the theoretical carbon residue of the mixture is $6.8 \%$, which is very close to the measured residue.

MS spectra of the thermal decomposition of the films show all the expected peaks of chitosan and lactic acid, accompanied with some extra peaks attributed to the presence of betaine. Peaks around $300{ }^{\circ} \mathrm{C}$ show that atomic and molecular oxygen are released, probably due to the decomposition of the carboxylic acid group. Another interesting peak is $m / z=40$, which could be attributed to $\mathrm{C}-\mathrm{CH}_{2}-\mathrm{N}^{+} \bullet$ due to the decomposition of betaine (Fig. S22, ESI $\dagger$ ).

TGA-MS of F/bet:LA mix. (Fig. S16, ESI $\dagger$ ) shows the same fragmentation pattern as F/bet:LA NaDES, without any major differences in the masses or the temperatures where they appear. TGA graphs, however, show some notable differences in the decomposition point $\left(T_{\text {onset }}=211{ }^{\circ} \mathrm{C}\right)$ and the carbon residue, which in this case is just $1 \%$. These differences are indications that the structures of the films are actually different and the presence of the NaDES has an active role in the formation and structure of the film, compared to the simple addition of the individual components in solution.

Similar to the previous case, the TGA F/ChCl:LA NaDES (Fig. S17, ESI $\dagger$ ) shows a significant shift in the decomposition point $\left(T_{\text {onset }}=225{ }^{\circ} \mathrm{C}\right)$ compared to ChCl:LA NaDES and F/AA. Specifically, this film shows the greatest resistance to thermal degradation of all the studied samples, as its decomposition point is higher than both individual components. The carbon residue is $5.1 \%$, again almost identical to the theoretical carbon residue of the mixture (calculated $5 \%$ ).

The MS spectra of the choline chloride containing films (F/ChCl:LA NaDES and F/ChCl:LA mix.) show the peaks for chitosan, with slight differentiations compared to F/bet:LA NaDES. In this case, oxygen release $(\mathrm{m} / \mathrm{z}=16$ and 32$)$ is insignificant, due to the presence of the hydroxyl group in choline chloride that decomposes in a different pathway compared to betaine. New peaks have appeared in this case, at $m / z=$ 15, 50 and 52. These could be attributed to $\mathrm{CH}_{3}{ }^{+\bullet}$ and $\mathrm{CH}_{3} \mathrm{Cl}$ released from the decomposition of choline chloride. The relative ratio of $m / z=50$ and 52 is exactly $76: 24$, which corresponds to the natural abundance of the two chlorine isotopes $\left({ }^{35} \mathrm{Cl}\right.$ and $\left.{ }^{37} \mathrm{Cl}\right)$. Thermal decomposition of ammonium chloride salts is widely studied and the nucleophilic elimination to alkyl chlorides is a generally accepted pathway. ${ }^{48,49}$

The TGA of F/ChCl:LA mix. (Fig. S18, ESI †) shows a lower decomposition point $\left(T_{\text {onset }}=209{ }^{\circ} \mathrm{C}\right)$ compared to F/ChCl:LA NaDES. More specifically, the decomposition point in this case is almost identical to that of the pure NaDES. The carbon residue follows the same trend as in the case of $F / b e t: L A$ NaDES, with the F/ChCl:LA mix. giving lower residue than expected (3.6 wt\%) The MS fragmentation pattern is identical to F/ChCl:LA NaDES, with no noteworthy differences. Similarly, the TGA spectra of the betaine and choline chloride (Fig. S22 and $\mathrm{S} 23$ respectively, ESI $\dagger$ ) indicate that the residue of betaine is higher than that of choline chloride.

Finally, as the water content is similar for the films containing the pre-formed NaDES or the corresponding mixture, it may be deduced that the slightly increased thickness observed in the former films (Table 2) is an inherent change, attributed to the polymer itself. A plausible explanation could be that the hydrogen bonding nature of the NADESs helps them permeate more between the chitosan chains, causing swelling of the polymer, which increases the volume of the film and, hence, its thickness. $^{50}$

\section{SEM}

In order to obtain further information on the morphology of the prepared films, SEM images were obtained (Fig. 8a-g). Interestingly, the morphology of the films appears to be different.

F/AA films present small holes with diameter of approximately $1.2 \mu \mathrm{m}$ possibly due to the evaporation of the acetic acid. On the other hand, the $\mathrm{F} /$ Tween presents a very wrinkled structure without any pores or holes.

The two NaDES containing films (F/bet:LA NaDES and F/ChCl:LA NaDES) appear to have a very different morphology compared to the other films. Their surface appears to be smooth with small wrinkled structured pores. These pores appear to be more frequent and larger in the F/bet:LA NaDES compared to those of the F/ChCl:LA NaDES sample. The lactic acid containing film does not present any holes in its surface, possibly because lactic acid does not evaporate. Finally, the F/ bet:LA mix. has a grain-like structure whereas the F/ChCl:LA mix. appears to have a wrinkled structured surface with pores.

SEM imaging revealed distinct differences among the films containing the NaDES or the corresponding mixture of the components. Comparing the F/bet:LA NaDES to the F/bet:LA mix. (Fig. 8c and f respectively), it can be observed that there is a uniformity in the surface of the former, while the latter has a less smooth surface. The same observation can be made when comparing the two choline chloride containing films.

The uniformity observed in the pre-formed NaDES containing films could be attributed to the existence some 'clusters' of the NaDES structure which, even though the dilution is high, 

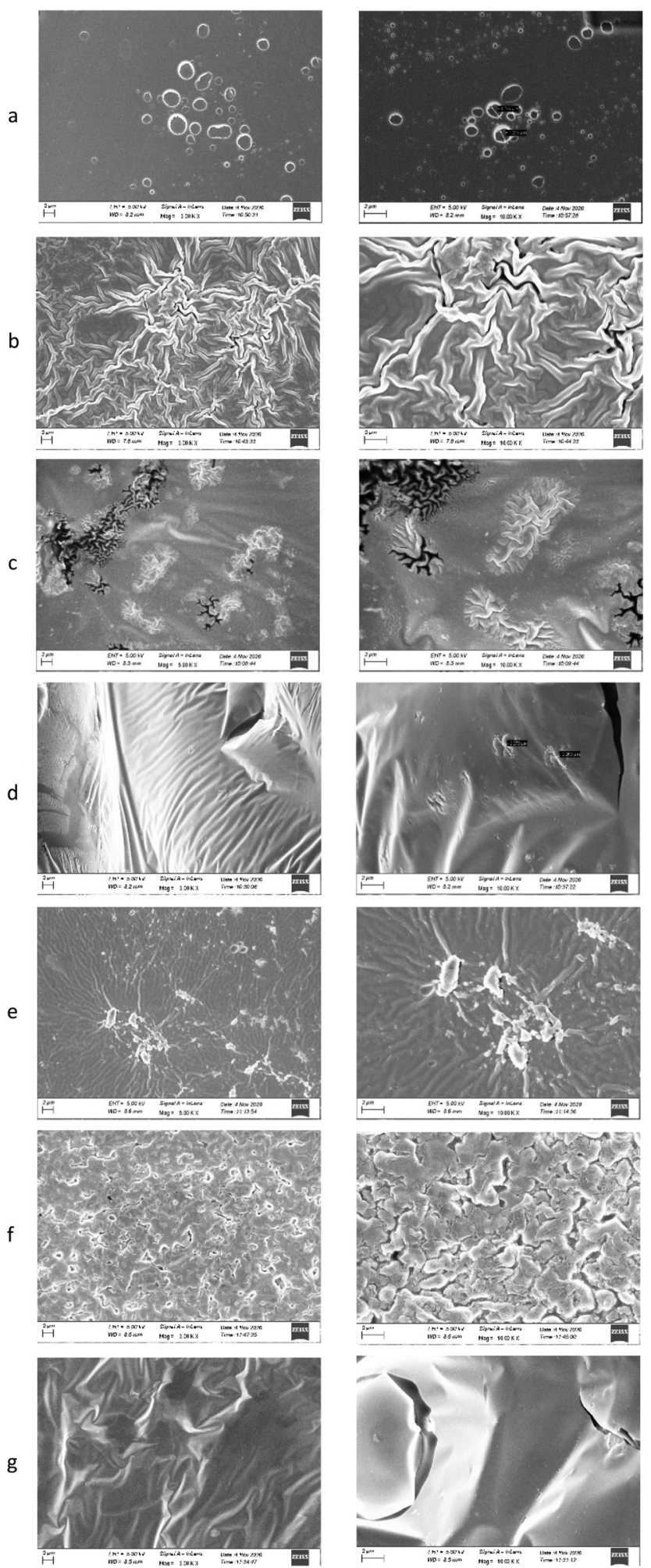

Fig. 8 SEM images of the (a) F/AA, (b) F/Tween, (c) F/bet:LA NaDES, (d) F/ChCl:LA NaDES, (e) F/0.5LA (f) F/bet:LA mix. and (g) F/ChCl:LA mix. Images on the left are at $5 \times 10^{3} \times$ magnification and on the right at $10^{4} \times$ magnification.

are maintained probably due to the interaction with the chitosan macromolecule.

\section{Experimental}

\section{Materials}

Chitosan 5-20 mPa s $\left(0.5 \%\right.$ in $0.5 \%$ acetic acid at $20{ }^{\circ} \mathrm{C}$, molecular weight $1526.464 \mathrm{~g} \mathrm{~mol}^{-1}$ ) was purchased from TCI, D,L-lactic acid $80-85 \%$ aq. soln., anhydrous betaine and choline chloride $98+\%$ were purchased from Alfa Aesar, and Tween 80 was from Aldrich.

All reagents were used without further purification. In all experiments, MilliQ (18.2 $\mathrm{M} \Omega$ at $25^{\circ} \mathrm{C}$ ) water was used.

\section{NaDES preparation}

The two NaDESs were prepared as described in the literature. Briefly, after choline chloride was dried in vacuo, lactic acid was added in molar ratio $1: 1.5$ for the synthesis of the ChCl:LA NaDES, ${ }^{30}$ while for the bet:LA NaDES the ratio was $1: 2 .^{31}$ In both cases, the synthesis took place at $50{ }^{\circ} \mathrm{C}$ under magnetic stirring until a viscous, colourless liquid was formed.

The $\mathrm{pH}$ of aqueous solutions of the two NaDESs, as well as the $\mathrm{pH}$ of the solution of the physical mixtures of their components, was measured with a Metrohm $744 \mathrm{pH}$ meter in order to identify the optimal concentration for the dissolution of chitosan (Fig. S1, ESI $\dagger$ ). Chitosan can only be dissolved in acidic aqueous solutions where its primary amino groups are protonated and, as a result, the polymer becomes a positively charged polyelectrolyte. ${ }^{32}$ Typically, chitosan is dissolved in solutions of acetic acid, hydrochloric acid, formic acid or lactic acid. Therefore, concentration $1 \% \mathrm{w} / \mathrm{v}$ of the two NaDESs was selected as the $\mathrm{pH}$ was 3 .

\section{Film preparation}

The casting method was employed for the preparation of the films. For each film, $0.2 \% \mathrm{w} / \mathrm{v}$ chitosan was left overnight at room temperature, under magnetic stirring, in order to be fully dissolved in the appropriate aqueous solvent. The solvents used are shown in Table 4 . Then, $45 \mathrm{~mL}$ of the film-forming solution was degassed and poured into crystallising dishes of base area of $25 \mathrm{~cm}^{2}$ and were left to dry in a Gallenkamp incubator for 3 days at $40{ }^{\circ} \mathrm{C}$. The films were peeled off the dish and stored in desiccator until testing. Each film was prepared in triplicate.

\section{Film characterisation}

Thickness. The thickness of the films was measured with a Hogetex digital micrometre $(0-25 \mathrm{~mm})$. Measurements were obtained in 5 different parts of each film.

Mechanical properties. The force required to rupture the film, referred as burst strength (BS) and total elongation of the film before breakage, referred as distance at burst (DB) of the films were measured with a TA.XT2i Texture Analyzer (Stable Micro System) equipped with a stainless steel penetration probe and analysed with the software Texture Exponent 32. The film was secured in a film supporting rig and the probe moved vertically towards the centre of the film at speed $1 \mathrm{~mm} \mathrm{~s}^{-1}$ until the film was ruptured.

Water vapour permeability (WVP). Water vapour permeability (WVP) was calculated following the Standard Test 
Table 4 Synthesis parameters of the prepared films

\begin{tabular}{|c|c|c|c|}
\hline Film & Component concentration (aq. solution) & Component concentration (\% w/v) & Plasticiser \\
\hline F/AA & $1 \% \mathrm{v} / \mathrm{v} \mathrm{AA}$ & $1.05 \% \mathrm{w} / \mathrm{v}$ & - \\
\hline F/Tween & $1 \% \mathrm{v} / \mathrm{v}$ AA & $1.05 \% \mathrm{w} / \mathrm{v}$ & $0.2 \% \mathrm{w} / \mathrm{v}$ Tween 80 \\
\hline F/bet:LA NaDES & 1\% w/v bet:LA NaDES & $1.00 \% \mathrm{w} / \mathrm{v}$ & - \\
\hline F/bet:LA mix. & 1\% w/v bet:LA mix. & $1.00 \% \mathrm{w} / \mathrm{V}$ & - \\
\hline $\mathrm{F} / 0.5 \mathrm{LA}$ & $0.5 \% \mathrm{v} / \mathrm{v} \mathrm{LA}$ & $0.61 \% \mathrm{w} / \mathrm{v}$ & - \\
\hline F/ChCl:LA NaDES & 1\% w/v ChCl:LA NaDES & $1.00 \% \mathrm{w} / \mathrm{v}$ & - \\
\hline F/ChCl:LA mix. & 1\% w/v ChCl:LA mix. & $1.00 \% \mathrm{w} / \mathrm{v}$ & - \\
\hline $\mathrm{F} / 0.4 \mathrm{LA}$ & $0.4 \% \mathrm{v} / \mathrm{v} \mathrm{LA}$ & $0.48 \% \mathrm{w} / \mathrm{v}$ & - \\
\hline
\end{tabular}

Method for Water Vapour Transmission of Materials. ${ }^{33,34}$ Briefly, each film was placed on top of a crystalising disk of bottom area $17.5 \mathrm{~cm}^{2}$, sealing the opening. The crystallisation disk contained $10 \mathrm{~mL}$ of water $(\mathrm{RH}=100 \%)$ and was placed in an equilibrated desiccator in which $\mathrm{RH}=0$. The mass loss of the system was periodically recorded over $24 \mathrm{~h}$, and plotted against time.

WVP was calculated using the following equation:

$$
W=(g / t A) \cdot(x / \Delta P)
$$

where: $g / t$ is the slope of mass against time $\left(q \mathrm{~s}^{-1}\right)$, A is the opening area in $m^{2}, x$ is the film's thickness $(m)$ and $\Delta P$ the partial vapour pressure (2338.8 $\mathrm{Pa}$ at $\left.20{ }^{\circ} \mathrm{C}\right)$.

For each film, three repetitions of the measurement were performed.

Fourier-transform infrared spectroscopy (FT-IR). FT-IR spectra of the films were measured using a Spectrum 100 by PerkinElmer, equipped with a Universal ATR (UATR) tool. The spectra are measured in 6 repeats and then averaged. The resolution of the spectra is $0.5 \mathrm{~cm}^{-1}$.

Thermogravimetric analysis (TGA). All TGA measurements were performed on a Mettler Toledo TGA/DSC 1 instrument, equipped with a HIDEN analytical HPRP20 - QIC evolved gas mass spectroscopy tool. 1-2 $\mathrm{mg}$ of film were placed in a weighed ceramic pan. The sample was heated to $50{ }^{\circ} \mathrm{C}$, were it remained for $15 \mathrm{~min}$ in order to equilibrate the balance and make sure the oven was filled with $\mathrm{N}_{2}$ and then heated to $700{ }^{\circ} \mathrm{C}$ with a rate of $5{ }^{\circ} \mathrm{C} \min ^{-1}$. The mass spec tool was adjusted to measure every $9 \mathrm{~s}$ and scan the mass range of $m / z 1$ to 300 , excluding 28 for $\mathrm{N}_{2}$, the molar mass of the carrier gas. In order to make the interpretation of the results easier, only the $\mathrm{m} / \mathrm{z}$ that showed a peak are presented.

Moisture content was calculated as the mass loss at $T=120{ }^{\circ} \mathrm{C}$ for all samples.

$T_{\text {onset: }}$ Temperature point where sample decomposition becomes significant. Calculated as the temperature point with $10 \%$ weight loss (after moisture loss).

$T_{\text {max.rate: }}$ Temperature of maximum decomposition rate.

$T_{1 / 2}$ : Temperature of $50 \%$ sample mass loss (taking into account the carbonaceous residue).

Scanning electron microscopy (SEM). The morphology of the films was identified using SEM analysis with a LEO 1525 Gemini FEG-SEM instrument (Zeiss, Oberkochen, Germany) at an applied voltage of $5 \mathrm{kV}$. The films were dried in vacuo and coated with chromium prior to the measurement.

\section{Conclusions}

In this work, we successfully prepared two chitosan-stabilised NaDES films and compared their properties with three conventionally prepared chitosan films and with the films arising from the addition of the NaDESs' components as an aqueous physical mixture.

Only little research on the field of chitosan dissolution directly into an aqueous solution of NaDES or their components $^{42,51}$ has been conducted and should be investigated further, as the low solubility of the polymer in a number of common solvents constitutes a major drawback. Our work was mainly focused on the preparation of the films and the comparison of their properties, therefore the full capacity of NaDESs' capability of dissolving chitosan is an aspect worthy of future investigation.

The obtained results suggest that the properties of the films can be easily adjusted to fit the requirements of a wide range of applications. The NaDES containing films or the films containing the physical mixture of the corresponding NaDES are very elastic and allow water to easily pass through. On the other hand, the films that do not contain a NaDES were inelastic and very resistant to rupture.

Additionally, the selection of NaDES also plays a vital role on the final properties of the films. The betaine containing films were twice as elastic as the choline chloride containing films, while the permeation of water through was only half. The presence of chitosan slightly affects the thermal properties of the films, increasing the indicative temperatures $T_{\text {onset }}, T_{\text {max.rate }}$ and $T_{1 / 2}$. SEM imaging revealed that the addition of a preformed NaDES, offers a uniformity on the morphology of the film, whereas, the separate addition of the two components is responsible for an uneven surface.

Our study indicates that the NaDES films are not completely identical to those produced from the direct mixture of NaDES's components. Indeed, the films appear to have almost identical DB and BS values, while the WVP, FTIR and thermal analysis reveal some minor differences. The mixture-films appear to be slightly better water vapour barriers and to decompose at slightly higher temperatures compared to the NaDES-films. Moreover, the FTIR of the produced films also appear to be non-identical. These results are consistent in our study, as they agree in both NaDES cases and are observed over multiple repetitions of our experiments. The observed differences are small, but statistically significant, and do not match our initial 
theory that the films should be completely identical regardless the production method. Therefore, further studies need to be conducted in order to completely understand the interactions in DES and how they are affected when they are diluted.

The aforementioned results demonstrate that the chitosanstabilised NaDES films are very promising alternatives for biomedical applications. Commercial wound healing patches and wound closure strips are mostly made of synthetic rubber enriched with bioactive compounds to promote healing, but usually lack in terms of elasticity. The prepared films are highly elastic, which allows the material to stretch with the skin's movements, which is significant for a safe and scar-free healing. On the other hand, the NaDESs used could be extracted and reused, leaving a porous material, consisting of pure chitosan with antibacterial properties. ${ }^{52}$ Moreover, the sustainable and biodegradable character of the product is of upmost importance for the reduction of health-care waste, which according the World Health Organisation has risen to approximately 1900 tonnes per year. ${ }^{53}$

\section{Author contributions}

Conceptualization A. R. N. P., A. D.; Data Curation A. R. N. P., S. K.; Methodology A. R. N. P., S. K.; Project Administration A. D.; Resources A. D., T. W.; Supervision A. D.; Writing - Original Draft Preparation A. R. N. P., S. K.; Writing - Review \& Editing, A. R. N. P., S. K., A. D., T. W.

\section{Conflicts of interest}

There are no conflicts to declare.

\section{Acknowledgements}

We thank Dr Lisa Haigh for training and providing S. K. with access to TGA-MS instrument of Imperial College Department of Chemistry's Mass Spec Suite. We thank Dr Mahmoud Ardakani for training and providing S. K. with access to Imperial College's Electron Microscopy Suite. A. R. N. Pontillo gratefully acknowledges financial support (scholarship for implementation of doctoral thesis) from the General Secretariat for Research and Technology (GSRT) and the Hellenic Foundation for Research and Innovation (HFRI).

\section{Notes and references}

1 A. Paiva, R. Craveiro, I. Aroso, M. Martins, R. L. Reis and A. R. C. Duarte, Natural Deep Eutectic Solvents - Solvents for the 21st Century, ACS Sustainable Chem. Eng., 2014, 2(5), 1063-1071.

2 Y. Dai, J. van Spronsen, G.-J. Witkamp, R. Verpoorte and Y. H. Choi, Natural deep eutectic solvents as new potential media for green technology, Anal. Chim. Acta, 2013, 766, 61.

3 Y. Liu, J. B. Friesen, J. B. McAlpine, D. C. Lankin, S.-N. Chen and G. F. Pauli, Natural deep eutectic solvents: properties, applications, and perspectives, J. Nat. Prod., 2018, 81(3), 679.

4 C. R. Ashworth, R. P. Matthews, T. Welton and P. A. Hunt, Doubly ionic hydrogen bond interactions within the choline chloride-urea deep eutectic solvent, Phys. Chem. Chem. Phys., 2016, 18(27), 18145.

5 D. Skarpalezos and A. Detsi, Deep eutectic solvents as extraction media for valuable flavonoids from natural sources, Appl. Sci., 2019, 9(19), 4169.

6 A. Tzani, C. Vaitsis, E. Kritsi, M. Smiljkovic, M. Sokovic, P. Zoumpoulakis and A. Detsi, Green synthesis of bis- $(\beta$ dicarbonyl)-methane derivatives and biological evaluation as putative anticandidial agents, J. Mol. Struct., 2020, 128276.

7 L. Cariati and R. M. Buoro, Evaluation of ionic natural deep eutectic solvents (NADES) modified binders towards the chemical properties of carbon paste electrodes, Electrochem. Commun., 2019, 109, 106605.

8 Y. Tian, Z. Zhu and D. W. Sun, Naturally sourced biosubstances for regulating freezing points in food researches: Fundamentals, current applications and future trends, Trends Food Sci. Technol., 2020, 95, 131.

9 D. I. Al-Risheq, M. S. Nasser, H. Qiblawey, I. A. Hussein and A. Benamor, Choline chloride based natural deep eutectic solvent for destabilization and separation of stable colloidal dispersions, Sep. Purif. Technol., 2020, 117737.

10 H. Xia, M. Ren, Y. Zou, S. Qin and C. Zeng, Novel Biocompatible Polysaccharide-Based Eutectogels with Tunable Rheological, Thermal, and Mechanical Properties: The Role of Water, Molecules, 2020, 25(15), 3314.

11 S. Marullo, A. Meli, N. T. Dintcheva, G. Infurna, C. Rizzo and F. D’Anna, Environmentally Friendly Eutectogels Comprising l-amino Acids and Deep Eutectic Solvents: Efficient Materials for Wastewater Treatment, ChemPlusChem, 2020, 85(2), 301.

12 A. R. N. Pontillo and A. Detsi, Nanoparticles for ocular drug delivery: modified and non-modified chitosan as a promising biocompatible carrier, Nanomedicine, 2019, 14(14), 1889.

13 A. Detsi, E. Kavetsou, I. Kostopoulou, I. Pitterou, A. R. N. Pontillo and A. Tzani, et al., Nanosystems for the encapsulation of natural products: The case of chitosan biopolymer as a matrix, Pharmaceutics, 2020, 12(7), 669.

14 D. Nataraj, S. Sakkara, M. Meghwal and N. Reddy, Crosslinked chitosan films with controllable properties for commercial applications, Int. J. Biol. Macromol., 2018, 120, 1256.

15 V. G. L. Souza, A. L. Fernando, J. R. A. Pires, P. F. Rodrigues, A. A. Lopes and F. M. B. Fernandes, Physical properties of chitosan films incorporated with natural antioxidants, Ind. Crops Prod., 2017, 107, 565.

16 S. Güneș and F. Tıhmınlığlu, Hypericum perforatum incorporated chitosan films as potential bioactive wound dressing material, J. Biol. Macromol., 2017, 102, 933.

17 S. Baghaie, M. T. Khorasani, A. Zarrabi and J. Moshtaghian, Wound healing properties of PVA/starch/chitosan hydrogel membranes with nano Zinc oxide as antibacterial wound dressing material, J. Biomater. Sci., Polym. Ed., 2017, 28(18), 2220 . 
18 Q. Li, F. Lu, G. Zhou, K. Yu, B. Lu and Y. Xiao, et al., Silver inlaid with gold nanoparticle/chitosan wound dressing enhances antibacterial activity and porosity, and promotes wound healing, Biomacromolecules, 2017, 18(11), 3766.

19 P. Cazón, M. Vázquez and G. Velazquez, Characterization of bacterial cellulose films combined with chitosan and polyvinyl alcohol: Evaluation of mechanical and barrier properties, Carbohydr. Polym., 2018, 195, 432.

20 A. Sangroniz, J.-B. Zhu, X. Tang, A. Etxeberria, E. Y.-X. Chen and H. Sardon, Packaging materials with desired mechanical and barrier properties and full chemical recyclability, Nat. Commun., 2019, 10(1), 1.

21 C. Caner, P. Vergano and J. Wiles, Chitosan film mechanical and permeation properties as affected by acid, plasticizer, and storage, J. Food Sci., 1998, 63(6), 1049.

22 A. S. S. Salazar, P. A. S. Cavazos, H. M. Paz and A. V. Fragoso, External factors and nanoparticles effect on water vapor permeability of pectin-based films, J. Food Eng., 2019, 245, 73.

23 A. Arora and G. Padua, Nanocomposites in food packaging, J. Food Sci., 2010, 75(1), R43.

24 L. W. McKeen, Permeability properties of plastics and elastomers, William Andrew, 2016.

25 M. Goh, Y. Hwang and G. Tae, Epidermal growth factor loaded heparin-based hydrogel sheet for skin wound healing, Carbohydr. Polym., 2016, 147, 251.

26 R. Xu, H. Xia, W. He, Z. Li, J. Zhao and B. Liu, et al., Controlled water vapor transmission rate promotes woundhealing via wound re-epithelialization and contraction enhancement, Sci. Rep., 2016, 6, 24596.

27 M. T. Khorasani, A. Joorabloo, A. Moghaddam, H. Shamsi and $\mathrm{Z}$. MansooriMoghadam, Incorporation of $\mathrm{ZnO}$ nanoparticles into heparinised polyvinyl alcohol/chitosan hydrogels for wound dressing application, Int. J. Biol. Macromol., 2018, 114, 1203-1215.

28 P. I. Morgado, A. Aguiar-Ricardo and I. J. Correia, Asymmetric membranes as ideal wound dressings: An overview on production methods, structure, properties and performance relationship, J. Membr. Sci., 2015, 490m, 139-151.

29 A. Gutiérrez, R. Alcalde, M. Atilhan and S. Aparicio, Insights on Betaine+ Lactic Acid Deep Eutectic Solvent, Ind. Eng. Chem. Res., 2020, 59(25), 11880-11892.

30 R. Alcalde, A. Gutiérrez, M. Atilhan and A. Aparicio, An experimental and theoretical investigation of the physicochemical properties on choline chloride-Lactic acid based natural deep eutectic solvent (NADES), J. Mol. Liq., 2019, 290, 110916.

31 V. I. Castro, F. Mano, R. L. Reis, A. Paiva and A. R. C. Duarte, Synthesis and physical and thermodynamic properties of lactic acid and malic acid-based natural deep eutectic solvents, J. Chem. Eng. Data, 2018, 63(7), 2548.

32 C. Pillai, W. Paul and C. P. Sharma, Chitin and chitosan polymers: Chemistry, solubility and fiber formation, Prog. Polym. Sci., 2009, 34(7), 641.

33 P. F. Pereira and C. T. Andrade, Optimized pH-responsive film based on a eutectic mixture-plasticized chitosan, Carbohydr. Polym., 2017, 165, 238.
34 Testing ASf, Materials. Standard test methods for water vapor transmission of materials: ASTM International, 2005.

35 A. Bégin and M.-R. Van Calsteren, Antimicrobial films produced from chitosan, Int. J. Biol. Macromol., 1999, 26(1), 63-67.

36 S. Prateepchanachai, W. Thakhiew, S. Devahastin and S. Soponronnarit, Mechanical properties improvement of chitosan films via the use of plasticizer, charge modifying agent and film solution homogenization, Carbohydr. Polym., 2017, 174, 253.

37 E. Jakubowska, M. Gierszewska, J. Nowaczyk and E. OlewnikKruszkowska, Physicochemical and storage properties of chitosan-based films plasticized with deep eutectic solvent, Food Hydrocolloids, 2020, 106007.

38 Q. F. Dang, J. Q. Yan, Y. Li, X. J. Cheng, C. S. Liu and X. G. Chen, Chitosan Acetate as an Active Coating Material and Its Effects on the Storing of Prunus avium L, J. Food Sci., 2010, 75(2), S125.

39 M. Matet, M.-C. Heuzey, E. Pollet, A. Ajji and L. Averous, Innovative thermoplastic chitosan obtained by thermomechanical mixing with polyol plasticizers, Carbohydr. Polym., 2013, 95(1), 241.

40 Y. Liu, J. Gu, J. Zhang, F. Yu, J. Wang and N. Nie, et al., $\mathrm{LiFePO}_{4}$ nanoparticles growth with preferential (010) face modulated by Tween-80, RSC Adv., 2015, 5(13), 9745.

41 A. Păucean, D. Vodnar, V. Mureşan, F. Fetea, F. Ranga and S. Man, et al., Monitoring lactic acid concentrations by infrared spectroscopy: A new developed method for Lactobacillus fermenting media with potential food applications, Acta Alimentaria, 2017, 46(4), 420.

42 M. A. Smirnov, A. L. Nikolaeva, V. K. Vorobiov, N. V. Bobrova, I. V. Abalov and A. V. Smirnov, et al., Ionic Conductivity and Structure of Chitosan Films Modified with Lactic Acid-Choline Chloride NADES, Polymers, 2020, 12(2), 350.

43 M. Francisco, A. van den Bruinhorst and M. C. Kroon, New natural and renewable low transition temperature mixtures (LTTMs): screening as solvents for lignocellulosic biomass processing, Green Chem., 2012, 14(8), 2153.

44 T. Altamash, M. S. Nasser, Y. Elhamarnah, M. Magzoub, R. Ullah and H. Qiblawey, et al., Gas solubility and rheological behavior study of betaine and alanine based natural deep eutectic solvents (NADES), J. Mol. Liq., 2018, 256, 286.

45 M. Viertorinne, J. Valkonen, I. Pitkänen, M. Mathlouthi and J. Nurmi, Keto-enol tautomerism, conformations and structure of $\mathrm{N}$-(2-hydroxy-5-methylphenyl), 2-hydroxybenzaldehydeimine, J. Mol. Struct., 1999, 477(1-3), 23.

46 N. Delgado-Mellado, M. Larriba, P. Navarro, V. Rigual, M. Ayuso and J. García, et al., Thermal stability of choline chloride deep eutectic solvents by TGA/FTIR-ATR analysis, J. Mol. Liq., 2018, 260, 37.

47 S. Koutsoukos, T. Tsiaka, A. Tzani, P. Zoumpoulakis and A. Detsi, Choline chloride and tartaric acid, a Natural Deep Eutectic Solvent for the efficient extraction of phenolic and carotenoid compounds, J. Cleaner Prod., 2019, 118384.

48 Y. Hao, J. Peng, S. Hu, J. Li and M. Zhai, Thermal decomposition of allyl-imidazolium-based ionic liquid studied by 
TGA-MS analysis and DFT calculations, Thermochim. Acta, 2010, 501(1-2), 78.

49 C. Maton, N. De Vos and C. V. Stevens, Ionic liquid thermal stabilities: decomposition mechanisms and analysis tools, Chem. Soc. Rev., 2013, 42(13), 5963.

50 A. Gugliuzza and E. Drioli, Carbon dioxide recovery from post-combustion processes: can gas permeation membranes compete with absorption, J. Membr. Sci., 2007, 300(1-2), 51.

51 M. A. Smirnov, A. L. Nikolaeva, V. K. Vorobiov, N. V. Bobrova, I. V. Abalov, A. V. Smirnov and M. P. Sokolova, Ionic conductivity and structure of chitosan films modified with lactic acid-choline chloride NADES, Polymers, 2020, 12(2), 350.

52 L. H. Delgado-Rangel, A. Huerta-Saquero, N. Eufracio-García, A. Meza-Villezcas, J. D. Mota-Morales and J. B. GonzálezCampos, Deep eutectic solvent-assisted phase separation in chitosan solutions for the production of 3D monoliths and films with tailored porosities, Int. J. Biol. Macromol., 2020, 164, 4084-4094.

53 Y. Chartier, Safe management of wastes from health-care activities, World Health Organization, 2014. 